\title{
Dose-finding study of intensive weekly alternating schedule of docetaxel, 5-fluorouracil, and oxaliplatin, FD/FOx regimen, in metastatic gastric cancer
}

\author{
Gemma Bruera ${ }^{1,2}$, Silvia Massacese ${ }^{3}$, Antonio Galvano ${ }^{4}$, Antonella Dal Mas ${ }^{5}$, \\ Stefano Guadagni, ${ }^{6,2}$, Giuseppe Calvisi ${ }^{5}$, Eugenio Ciacco ${ }^{3}$, Antonio Russo ${ }^{4}$, Enrico \\ Ricevuto $^{1,2}$, on behalf of Oncology Network ASL1 Abruzzo, Italy \\ ${ }^{1}$ Oncology Territorial Care, S. Salvatore Hospital, Oncology Network ASL1 Abruzzo, University of L'Aquila, L'Aquila, Italy \\ ${ }^{2}$ Department of Biotechnological and Applied Clinical Sciences, University of L'Aquila, L'Aquila, Italy \\ ${ }^{3}$ Pharmacy Unit, S. Salvatore Hospital, Oncology Network ASL1 Abruzzo, L'Aquila, Italy \\ ${ }^{4}$ Medical Oncology, Department of Surgical, Oncological and Stomatological Sciences, University of Palermo, Palermo, Italy \\ ${ }^{5}$ Pathology, S. Salvatore Hospital, Oncology Network ASL1 Abruzzo, L'Aquila, Italy \\ ${ }^{6}$ University General Surgery, S. Salvatore Hospital, Oncology Network ASL1 Abruzzo, University of L'Aquila, L'Aquila, Italy \\ Correspondence to: Antonio Russo, email: antonio.russo@usa.net
}

Keywords: docetaxel, 5-fluorouracil, and oxaliplatin; FD/FOx intensive regimen; dose-finding study; first-line triplet chemotherapy; metastatic gastric cancer

Received: January 24, $2018 \quad$ Accepted: March 07, $2018 \quad$ Published: April 17, 2018

Copyright: Bruera et al. This is an open-access article distributed under the terms of the Creative Commons Attribution License 3.0 (CC BY 3.0), which permits unrestricted use, distribution, and reproduction in any medium, provided the original author and source are credited.

\section{ABSTRACT}

Introduction: Proper administration timing, dose-intensity, efficacy/toxicity ratio of triplet docetaxel (DTX), 5-fluorouracil (5-FU), and oxaliplatin (OXP) should be improved to safely perform three-drugs intensive first line in advanced gastric cancer (GC). This dose-finding study investigated recommended 5-FU and OXP doses, safety of triplet regimen and preliminary activity.

Methods: Schedule: $12 \mathrm{~h}$-timed-flat-infusion 5-FU 700-1000 mg/m² $/ \mathrm{d} \mathrm{1-2}^{2}$ 8-9, $15-16,22-23$, with $100 \mathrm{mg} / \mathrm{m}^{2} / \mathrm{d}$ increase for dose level; DTX $50 \mathrm{mg} / \mathrm{m}^{2} \mathrm{~d} \mathrm{1}, 15$ fixed dose, OXP at three increasing dose-levels $60-70-80 \mathrm{mg} / \mathrm{m}^{2} \mathrm{~d} 8,22$, every 4 weeks. Intra- and inter-patients dose-escalation was planned.

Results: Ten fit $<75$ years patients were enrolled: median age 59; young-elderly 4 $(40 \%)$. From first to fifth dose level, 5 patients ( 1 per cohort) were enrolled according to intra-patient dose escalation, no dose-limiting toxicity (DLT) were reported. At sixth level, 1 DLT, G2 diarrhea, was reported, thus other 2 patients were enrolled, DLT $1 / 3$ patients (33\%). Maximum tolerated dose (MTD) was not reached. 5-FU and OXP recommended doses (RD) were $1000 \mathrm{mg} / \mathrm{m}^{2} / \mathrm{d}$ and $80 \mathrm{mg} / \mathrm{m}^{2}$, respectively. To confirm RD, other 3 patients were enrolled, without DLT. Cumulative G3-4 toxicities were: neutropenia $50 \%$, leucopenia $20 \%$, hypoalbuminemia $10 \%$, mucositis $10 \%$, asthenia $20 \%$. Limiting toxicity syndromes were $30 \%, 25 \%$ in young-elderly, all multiple site. Objective response rate intent-to-treat $60 \%$, disease control rate $90 \%$. After 15 months follow-up, progression-free and overall survival, 6 and 17 months, respectively.

Conclusions: First line intensive FD/FOx regimen adding DXT/5-FU/OXP can be safely administered at recommended doses in advanced GC, with promising high activity and efficacy. 


\section{INTRODUCTION}

Clinical management of advanced gastric cancer (GC) faces with different options of medical treatment strategies according to patients' fitness (age, performance status (PS), comorbidities), extension of primary tumor and related symptoms, influencing nutritional and functional conditions. Over the last 40 years, different active drugs were evaluated, and used in mono, doublet, and triplet chemotherapy combinations, improving activity, quality of life (QoL), and overall survival (OS). 5 -fluorouracil (5FU)-based regimens consisting of mono, doublet or triplet combinations of chemotherapeutic drugs, improved activity and efficacy of first line advanced or metastatic GC.

5-FU, doxorubicin plus mitomycin (FAM) and 5-FU plus doxorubicin (Adriamycin) (FA) did not significantly increased $\mathrm{OS}$ vs 5-FU alone [1]. Epirubicin, cisplatin, and 5-FU (ECF) vs FAMTX significantly improved survival and QoL: overall response rate (ORR) 45 vs $21 \%$, progression-free survival (PFS) 7.4 vs 3.4 months, OS 8.9 vs 5.7 months [2]. Mitomycin, cisplatin, and protracted venous-infusion (PVI) 5-FU, MCF regimen, compared with ECF, did not significantly increased outcomes: ORR 44.1 vs $42.4 \%$, PFS 7 months for both regimens, OS 8.7 vs 9.4 months; QoL was better with ECF [3].

Doublet 5-FU leucovorin/OXP (FLO) reported ORR $44.9 \%$, PFS 6.2 months, OS 8.6 months [4]. A trend toward improved PFS 5.8 vs 3.9 months, with no significantly different OS (10.7 vs 8.8 months), was reported with FLO association. [5]. In elderly $>65$ years patients, FLO resulted in significantly increased ORR (41.3 vs $16.7 \%)$, time to treatment failure (5.4 vs 2.3 months), PFS (6.0 vs 3.1 months), and improved OS (13.9 vs 7.2 months).

In REAL2 trial, patients received triplet with epirubicin and cisplatin plus either 5-FU (ECF) or capecitabine (ECX) or triplet with epirubicin and OXP plus either 5-FU (EOF) or capecitabine (EOX) [6]: OS were 9.9, 9.9, 9.3, 11.2 months, respectively, significantly longer with EOX vs ECF; PFS and ORR not significantly different. In ML17032 trial, cisplatin plus capecitabine (XP) compared to cisplatin plus 5-FU (FP) demonstrated: ORR 41 vs $29 \%$, PFS 5.6 vs 5.0 months, OS 10.5 vs 9.3 months [7]. OS and ORR were superior with capecitabinevs 5-FU-based combinations [8], PFS not significantly different in a meta-analysis of these two randomized phase III trials.

Major challenge of more drugs addition in a chemotherapy regimen is designing proper schedule and doses, providing the adequate balance between dose intensity (DI) of each drug and safety. DTX addiction to cisplatin/5-FU-based triplet chemotherapy regimen was evaluated in randomized studies [9, 10], at different doses and schedules, with significantly increased toxicity, limiting the favourable impact and expected efficacy of the association. In phase II/III V325 trial, DCF significantly increased outcomes compared with CF [9]: ORR 37 vs $25 \%, P 0.01$, PFS 5.6 vs 3.7 months, $P<0.001$, and OS 9.2 vs 8.6 months, $P 0.02$.

Modulated DCF regimen demonstrated efficacy and improved safety profile in different clinical trials [11-15]. FLOT schedule reported in a phase II trial ORR 57.7\%, PFS 5.2 months, OS 11.1 months [11]. Outcomes were not significantly different with DCF compared with modified DCF (mDCF) in a retrospective evaluation [12]: ORR 45.6 vs $46.7 \%, P>0.05$, PFS and OS 7.4 vs $6.5,9.9$ vs 8.6 months $P>0.05$. In a phase II randomized trial, DTX/ $\mathrm{OXP} / 5$-FU reported higher ORR and longer PFS and OS compared with DTX/OXP, and DTX/OXP/capecitabine: $46.6 \%, 7.66$ months, 14.59 months [14]. First line DOC triplet association [13], reported ORR 52.1\%, PFS and OS 6.9 and 12.6 months, respectively. In another randomized phase II study, mDCF regimen improved efficacy compared with DCF [15]: 6-month PFS 63 vs $53 \%$, median OS 18.8 vs 12.6 months, $P 0.007$.

More, different clinical trials evaluated irinotecan (CPT-11) in advanced GC. In a phase III randomized trial, CPT-11/5-FU was not inferior to cisplatin/5-FU, OS 9.0 vs 8.7 months, ORR 31.8 vs $25.8 \%$ [16]. In a randomized phase II trial, capecitabine was added to CPT-11 or cisplatin: ORR 37.7 vs $42.0 \%$, PFS 4.2 vs 4.8 months, with trend toward better OS 10.2 vs 7.9 months [17].

In French randomized trail comparing first line epirubicin, cisplatin, and capecitabine (ECX) with 5-FU, leucovorin, and CPT-11 (FOLFIRI), with predefined second line (FOLFIRI for ECX, ECX for FOLFIRI arm), time to failure was significantly longer with FOLFIRI than ECX (5.1 vs 4.2 months, $P$ 0.008) [18].

More recently, in phase III randomized SPIRITS trial, S-1 plus cisplatin was compared with S-1 [19]. OS and PFS were significantly longer in S-1/cisplatin, 13.0 vs 11.0 months, $P 0.04$, and 6.0 vs 4.0 months, $P<0.0001$. In western population, cisplatin and S-1 determined ORR $51 \%$, median OS 10.9 months [20]. In phase III FirstLine Advanced Gastric Cancer Study (FLAGS), S-1 plus cisplatin resulted in similar OS compared with 5-FU plus cisplatin: 8.6 vs 7.9 months $[21,22]$. In a randomized phase 3 trial, OS was statistically non-inferior with S-1/ cisplatin compared to 5 -FU/cisplatin (8.6 vs 7.9 months) [23].

We previously developed, in metastatic colorectal cancer setting, clinical trials proposing the concept of alternating chemotherapy administration schedules, as well as $12 \mathrm{~h}$-timed-flat-infusion (TFI)/5-FU administration modality, to optimize safety profile and maintain adequate dose intensity of each drug, in order to intensify first line regimens in fit patients [24-29]. The present dose-finding study, proposing intensive triplet chemotherapy combining first line DXT/OXP/5-FU in metastatic GC patients, assess $\mathrm{OXP}$ and 5-FU doses to be recommended in association with DXT for prospective clinical trials, safety and preliminary activity. 


\section{RESULTS}

\section{Patient demographics}

Ten consecutive patients were enrolled (Table 1): Male/Female ratio, 6/4; median age, 59 years; 4 (40\%) young-elderly $(\geq 65<75 \mathrm{y})$; CIRS stage primary $6(60 \%)$, intermediate $4(40 \%)$ [30]; WHO PS $08(80 \%), 1-22$ $(20 \%)$; primary tumor location, antrum $2(20 \%)$, body $6(60 \%)$, fundus $1(10 \%)$, cardiac $1(10 \%)$. Metastatic disease synchronous in all patients. Metastatic sites: liver 7 patients (70\%), lung 4 (40\%), lymph nodes 4 (40\%), local recurrence 7 (70\%), peritoneal carcinomatosis $3(30 \%)$. Metastatic site was single in 1 patient $(10 \%)$ with liver metastates, multiple in 9 patients $(90 \%)$. Liver metastases were all multiple.

\section{Dose finding}

DTX was administered at $50 \mathrm{mg} / \mathrm{m}^{2}$ fixed dose (Table 2). At the first dose-level (5-FU $700 \mathrm{mg} / \mathrm{m}^{2} / \mathrm{d}$ and OXP $60 \mathrm{mg} / \mathrm{m}^{2}$ ), 1 patient was enrolled and 2 cycles of treatment were administered; no dose-limiting toxicity (DLT) was observed. At the second dose level (5-FU 800 $\mathrm{mg} / \mathrm{m}^{2} / \mathrm{d}$ and OXP $60 \mathrm{mg} / \mathrm{m}^{2}$ ), 2 patients (1 new patient) were treated, and 4 cycles of treatment were administered; no DLT was observed. At the third dose level (5-FU 900 $\mathrm{mg} / \mathrm{m}^{2} / \mathrm{d}$ and OXP $60 \mathrm{mg} / \mathrm{m}^{2}$ ), 3 patients (1 new patient) were treated, and 5 cycles of treatment were administered; no DLT was observed. At the fourth dose level (5-FU 900 $\mathrm{mg} / \mathrm{m}^{2} / \mathrm{d}$ and OXP $70 \mathrm{mg} / \mathrm{m}^{2}$ ), 3 patients (1 new patient) were treated, and 4 cycles of treatment were administered; no DLT was observed. At the fifth dose level (5-FU 900 $\mathrm{mg} / \mathrm{m}^{2} / \mathrm{d}$ and OXP $80 \mathrm{mg} / \mathrm{m}^{2}$ ), 6 patients (1 new patient) were treated, and 9 cycles of treatment were administered; no DLT was observed. At sixth dose level (5-FU $1000 \mathrm{mg} /$ $\mathrm{m}^{2} / \mathrm{d}$ and OXP $\left.80 \mathrm{mg} / \mathrm{m}^{2}\right), 9$ patients (5 new patients) were treated, and 22 cycles were administered. A DLT, G2 diarrhea, was observed in 1 patient (no new patient), then other 2 patients were enrolled at this dose level without other DLT, 1 out of 3 patients (33\%) of the first cohort. Thus, maximum tolerated dose (MTD) was not reached, and the recommended doses of the association was $12 \mathrm{~h}-\mathrm{TFI} / 5-\mathrm{FU} 1000 \mathrm{mg} / \mathrm{m}^{2} / \mathrm{d} \mathrm{d} 1-2,8-9,15-16,22-23$; DTX $50 \mathrm{mg} / \mathrm{m}^{2} \mathrm{~d} 1,15$; OXP $80 \mathrm{mg} / \mathrm{m}^{2} \mathrm{~d} 8$, 22. In order to confirm the recommended doses, other 3 patients were enrolled at this dose-level, without other DLT. Thus, 1 DLT was observed out of 9 patients (11\%) and out of 22 cycles of treatment $(4 \%)$.

\section{Dose-intensity}

Median number of administered cycles of treatment was 5.5 (range 3-8). Median received dose intensity (rDI) per patient were: DTX $22.75 \mathrm{mg} / \mathrm{m}^{2} /$ week $(91 \%$ of recommended-DI), OXP $33.5 \mathrm{mg} / \mathrm{m}^{2} /$ week $(83.75 \%)$,
5 -FU $1657.5 \mathrm{mg} / \mathrm{m}^{2} /$ week (82.87\%), respectively. In 4 young-elderly patients, median rDIs per patient were: DTX $21.25 \mathrm{mg} / \mathrm{m}^{2} /$ week ( $85 \%$ of recommended-DI), OXP $29.5 \mathrm{mg} / \mathrm{m}^{2} /$ week (73.75\%), 5-FU $1513.75 \mathrm{mg} / \mathrm{m}^{2} /$ week $(75.68 \%)$, respectively.

\section{Toxicity}

Table 3 describes cumulative toxicities in 10 enrolled patients and in 50 administered cycles. No patient discontinued treatment due to limiting toxicity. Cumulative G3-4 toxicities, by patients were: neutropenia $50 \%$, leucopenia $20 \%$, hypoalbuminemia $10 \%$, mucositis $10 \%$, asthenia $20 \%$. Limiting toxicities were reported in 1 out of $4(25 \%)$ young-elderly patients: G3 hypoalbuminemia, G3 mucositis. G2 toxicities by patients were: diarrhea $40 \%$, asthenia $60 \%$, hypoalbuminemia $30 \%$, neurotoxicity $30 \%$, anemia $10 \%$, thrombocytopenia $10 \%$. No case of thrombosis, hemorrhage/bleeding, cardiac or cerebrovascular ischemia, G3-4 thrombocytopenia, or toxic deaths were observed.

Overall, limiting toxicity syndromes (LTS) [25, 28], all multiple sites (LTS-ms), were observed in 3 patients (30\%): 1 out of 4 young-elderly patients (25\%), characterized by G3 hypoalbuminemia, G3 mucositis, and G2 neurotoxicity, associated with G2 asthenia, G3 neutropenia; 2 out of 6 non-elderly patients $(33.3 \%)$, characterized by G3 asthenia, G2 neurotoxicity and G4 leuconeutropenia, associated with G2 diarrhea, G2 anorexia, G2 alopecia, in 1 patient, and by G3 asthenia and $\mathrm{G} 2$ neurotoxicity, associated with G2 constipation, G2 diarrhea, G2 mucositis, G3 neutropenia, in 1 patient.

\section{Activity and efficacy}

Overall, 10 patients were enrolled (Table 4), 7 treated in the dose escalation phase and 3 at the recommended doses. In the intent-to-treat and as-treated analyses all 10 patients were evaluable: ORR was $60 \%$ $(\alpha 0.05, \mathrm{CI} \pm 32)$. We observed 1 complete and 5 partial responses, 3 stable disease and 1 progression. Disease Control Rate (DCR) was $90 \%(\alpha 0.05, \mathrm{CI} \pm 19)$. After a median follow-up of 15 months, median PFS was 6 months (3-15): 9 events occurred. Median OS was 17 months $(5+-26): 7$ events occurred. Secondary resection of liver metastasis was performed in 1 patient with PFS of 10 months, and clinical complete response after triplet chemotherapy regimen.

Second line treatments were CPT-11 or ramucirumab-based chemotherapy associations in suitable patients.

\section{DISCUSSION}

One of the major reason that can justify the failure of the association of DTX to doublet platinum/5-FU to 


\begin{tabular}{|c|c|}
\hline \multirow{2}{*}{ No. of patients } & Total N. (\%) \\
\hline & 10 \\
\hline $\begin{array}{l}\text { Sex } \\
\quad \text { Male/Female }\end{array}$ & $6 / 4$ \\
\hline $\begin{array}{l}\text { Age, years } \\
\text { median } \\
\text { range } \\
\geq 65 \text { years }\end{array}$ & $\begin{array}{c}59 \\
42-73 \\
4(40)\end{array}$ \\
\hline $\begin{array}{l}\text { CIRS stage } \\
\text { primary } \\
\text { intermediate } \\
\text { secondary }\end{array}$ & $\begin{array}{l}6(60) \\
4(40) \\
-\end{array}$ \\
\hline $\begin{array}{l}\text { WHO Performance Status } \\
0 \\
1-2\end{array}$ & $\begin{array}{l}8(80) \\
2(20)\end{array}$ \\
\hline $\begin{array}{l}\text { Metastatic disease } \\
\text { synchronous } \\
\text { metachronous }\end{array}$ & $\begin{array}{c}10(100) \\
-\end{array}$ \\
\hline $\begin{array}{l}\text { Primary tumor location } \\
\text { antrum } \\
\text { body } \\
\text { fundus } \\
\text { cardias }\end{array}$ & $\begin{array}{l}2(20) \\
6(60) \\
1(10) \\
1(10)\end{array}$ \\
\hline $\begin{array}{l}\text { No. of involved sites } \\
\quad 1 \\
\quad \geq 2\end{array}$ & $\begin{array}{l}1(10) \\
9(90)\end{array}$ \\
\hline $\begin{array}{l}\text { Sites of metastases } \\
\text { liver } \\
\text { lung } \\
\text { lymph nodes } \\
\text { local recurrence } \\
\text { peritoneal carcinomatosis }\end{array}$ & $\begin{array}{l}7(70) \\
4(40) \\
4(40) \\
7(70) \\
3(30)\end{array}$ \\
\hline
\end{tabular}

Abbreviation: CIRS, Cumulative Illness Rating Scale; WHO, World Health Organization.

significantly increase activity and efficacy of metastatic GC patients in clinical practice can be ascribed to toxicity limiting the effective realization of this intensive first line chemotherapeutic strategy. The present dose-finding study proposing DTX, 5-FU and OXP triplet chemotherapy association, recommended 5 -FU dose $1000 \mathrm{mg} / \mathrm{m}^{2} / \mathrm{d}$ and OXP $80 \mathrm{mg} / \mathrm{m}^{2}$ for safely administration in clinical trials to properly evaluate their contribution to clinical outcome as first line treatment in metastatic GC patients. FD/FOx association was feasible at median DTX rDI 92\% (23 mg/ $\mathrm{m}^{2} /$ week), 5-FU rDI $83 \%$ (1657 mg/ $\mathrm{m}^{2} /$ week), and OXP rDI $92 \%$ ( $23 \mathrm{mg} / \mathrm{m}^{2} /$ week $)$. Cumulative G3-4 toxicities were represented by neutropenia $50 \%$, leucopenia $20 \%$, hypoalbuminemia $10 \%$, mucositis $10 \%$, asthenia $20 \%$. Three individual LTS, all LTS-ms, were reported in 30\% patients. G2 toxicities were represented by diarrhea $40 \%$, asthenia $60 \%$, hypoalbuminemia $30 \%$, neurotoxicity $30 \%$, anemia $10 \%$, thrombocytopenia $10 \%$.

Major features of the proposed schedule are: 12 hours nightly-TFI infusion 5-FU limiting FU-related side effects, specifically mucositis and diarrhea, compared to all combinations containing 5-FU, and weekly alternating infusion of DTX and OXP, modulating intensity of the concomitant infusion of triplet drugs every 3 weeks. To this concern, it may limit specifically reported toxicity: mucositis and diarrhea related to 5-FU alone; anorexia, nausea, vomiting, leucopenia, thrombocytopenia with FAM [1]; haematological with FAMTX [2]; G3-4 neutropenia with ECF, and thrombocytopenia and handfoot syndrome with MCF [3]. Grade 3-4 toxicities with 
Table 2: 5-fluorouracil and oxaliplatin dose-finding

\begin{tabular}{|c|c|c|c|c|c|c|c|}
\hline $\begin{array}{l}\text { Dose } \\
\text { levels }\end{array}$ & $\begin{array}{c}\text { docetaxel }\left(\mathrm{mg} / \mathrm{m}^{2} \mathrm{~d} 1,15\right)- \\
\text { oxaliplatin }\left(\mathrm{mg} / \mathrm{m}^{2} \mathrm{d8}, 22\right) \\
5 \text {-fluorouracil }\left(\mathrm{mg} / \mathrm{m}^{2} / \mathrm{d}\right. \\
\text { d1-2,8-9,15-16,22-23) }\end{array}$ & $\begin{array}{l}\text { No. patients } \\
\text { (new } \\
\text { patients) }^{\mathrm{a}}\end{array}$ & $\begin{array}{l}\text { No. } \\
\text { cycles }\end{array}$ & $\begin{array}{c}\text { No. patients } \\
\text { with DLT/ total } \\
\text { patients }(\%)\end{array}$ & $\begin{array}{l}\text { No. patients } \\
\text { with DLT/ new } \\
\text { patients (\%) }\end{array}$ & $\begin{array}{l}\text { No. cycles with } \\
\text { DLT/ total } \\
\text { cycles }(\%)\end{array}$ & DLTs \\
\hline I & $50-60-700$ & $1(1)$ & 2 & $0 / 1(0)$ & $0 / 1(0)$ & $0 / 2(0)$ & - \\
\hline II & $50-60-800$ & $2(1)$ & 4 & $0 / 2(0)$ & $0 / 1(0)$ & $0 / 4(0)$ & - \\
\hline III & $50-60-900$ & $3(1)$ & 5 & $0 / 3(0)$ & $0 / 1(0)$ & $0 / 5(0)$ & - \\
\hline IV & $50-70-900$ & $3(1)$ & 4 & $0 / 3(0)$ & $0 / 1(0)$ & $0 / 4(0)$ & - \\
\hline V & $50-80-900$ & $6(1)$ & 9 & $0 / 6(0)$ & $0 / 1(0)$ & $0 / 9(0)$ & - \\
\hline VI & $50-80-1000$ & $9(5)$ & 22 & 1/9 (11) & $0 / 5(0)$ & $1 / 22(4)$ & $\begin{array}{c}\text { G2 } \\
\text { diarrhea }\end{array}$ \\
\hline
\end{tabular}

a intra- and inter-patients dose escalation.

Abbreviation: DLT, dose-limiting toxicity.

FLO regimen were: neutropenia $38 \%$, thrombocytopenia $4 \%$, anemia $11 \%$, neurotoxicity $21 \%$ [4]. Cumulative toxicity reported with FLO regimen, $24 \mathrm{~h}$ infusion 5 -FU $2600 \mathrm{mg} / \mathrm{m}^{2}$, leucovorin $200 \mathrm{mg} / \mathrm{m}^{2}$, and OXP $85 \mathrm{mg} /$ $\mathrm{m}^{2}$, compared with $24 \mathrm{~h}$ infusion $5-\mathrm{FU} 2000 \mathrm{mg} / \mathrm{m}^{2}$, leucovorin $200 \mathrm{mg} / \mathrm{m}^{2}$, and cisplatin $50 \mathrm{mg} / \mathrm{m}^{2}$, every 2 weeks, was favourable: anemia (54 vs $72 \%$ ), nausea (53 vs $70 \%$ ), vomiting (31 vs $52 \%$ ), alopecia ( 22 vs $39 \%$ ), asthenia (19 vs 34\%), neurotoxicity (22 vs 63\%) [5]. In REAL2 trial, safety profile of capecitabine and 5-FU were similar; lower incidences of G3-4 neutropenia, alopecia, renal toxicity, but slightly higher incidences of diarrhea and neuropathy were associated with OXP compared with cisplatin [6]. In ML17032 trial, prevalent G3-4 toxicities in XP vs FP were: neutropenia (16 vs 19\%), vomiting (7 vs $8 \%$ ), stomatitis (2 vs 6\%) [7].

Safety of DTX addiction to cisplatin/5-FU in a triplet chemotherapy regimen was evaluated in different clinical studies $[9,10]$.

Our preliminary data concerning activity and efficacy show that the present schedule of safely administration of DTX/5-FU/OXP association may achieve $60 \%$ ORR, 90\% DCR, median PFS 6 months, median OS 17 months. ECF regimens reported ORR 44.145\%, PFS 7-7.4 months, OS 8.7-8.9 months [2, 3]; FLO association reported ORR 44.9\%, PFS 5.8-6.2 months, OS 8.6 months $[4,5]$, particularly relevant in elderly patients, ORR 41.3\%, PFS 6.0 months, OS 13.9 months [5]; EOX regimen reached 11.2 months OS [7]. In fit patients, intensive regimens adding DTX to cisplatin/5-FU reported ORR ranging between 37-46.7\%, PFS 5.6-9.2 months, OS 8.6-12.6 months $[5,9,12,15]$. In V325 trial, patients were randomized to DXT $75 \mathrm{mg} / \mathrm{m}^{2}$ and cisplatin $75 \mathrm{mg} /$ $\mathrm{m}^{2} \mathrm{~d} 1$ plus 5 -FU $750 \mathrm{mg} / \mathrm{m}^{2} / \mathrm{d} \mathrm{d} 1-5$, every 3 weeks (DCF) or cisplatin $100 \mathrm{mg} / \mathrm{m}^{2} \mathrm{~d} 1$ plus $5-\mathrm{FU} 1000 \mathrm{mg} / \mathrm{m}^{2} / \mathrm{d} \mathrm{d} 1-5$, every 4 weeks (CF) [9]. Prevalent G3-4 toxicities in DCF vs CF arms were: neutropenia ( 82 vs 57\%), stomatitis (21 vs $27 \%$ ), diarrhea (19 vs $8 \%$ ), asthenia (19 vs $14 \%$ ).

Different clinical trials evaluated modulated DCF regimen and demonstrated improved safety profile [1115]. Modified DCF regimen reported ORR 45.6\%, PFS 7.4 months, OS 9.9-18.8 months $[12,15]$. In a retrospective evaluation DCF, $75 \mathrm{mg} / \mathrm{m}^{2}$ DTX and cisplatin d1 plus 5 -FU $750 \mathrm{mg} / \mathrm{m}^{2} /$ day d $1-5$, every 3 weeks, was compared with modified DCF (mDCF), including $60 \mathrm{mg} / \mathrm{m}^{2}$ DTX and cisplatin $\mathrm{d} 1$ and $5-\mathrm{FU} 600 \mathrm{mg} / \mathrm{m}^{2}$ continuous infusion d1-5, every 3 weeks [12]. Prevalent G3-4 toxicities were higher in DCF arm: neutropenia (48.2 vs $13.6 \% P 0.003)$, anemia (21.2 vs $4.5 \% P 0.06)$, nausea (44.7 vs $13.6 \%$ $P 0.008)$, and vomiting (31.8 vs $4.5 \% P 0.01) . \mathrm{mDCF}$ regimen (48-h infusion 5-FU $2000 \mathrm{mg} / \mathrm{m}^{2}$, DTX $40 \mathrm{mg} / \mathrm{m}^{2}$ $\mathrm{d} 1$, cisplatin $40 \mathrm{mg} / \mathrm{m}^{2} \mathrm{~d} 3$, every 2 weeks) compared with DCF (DXT $75 \mathrm{mg} / \mathrm{m}^{2}$, cisplatin $75 \mathrm{mg} / \mathrm{m}^{2}$, and 5-FU 750 $\mathrm{mg} / \mathrm{m}^{2} \mathrm{IV}$ over 5 days with granulocyte colony-stimulating factor, every 3 weeks) [15], reported 76\% G3-4 toxicities in $\mathrm{mDCF}$ arm; DCF arm closed early because of $90 \%$ limiting toxicities.

Addition of DTX to OXP and 5-FU- or capecitabinebased first line chemotherapy represents a step forward to intensify medical treatment of GC patients, maintaining good tolerability: ORR 46.6-57.7\%, PFS 5.2-7.6 months, OS 11.1-14.5 months [11, 14]. Prevalent G3-4 toxicities reported with FLOT schedule, biweekly DTX $50 \mathrm{mg} / \mathrm{m}^{2}$, OXP $85 \mathrm{mg} / \mathrm{m}^{2}$, and 24-h infusion 5-FU $2600 \mathrm{mg} / \mathrm{m}^{2}$ plus leucovorin $200 \mathrm{mg} / \mathrm{m}^{2}$, were: neutropenia $48.1 \%$, leucopenia $27.8 \%$, diarrhea $14.8 \%$, asthenia $11.1 \%$ [11]. Better safety profile was reported with DTX/OXP/5-FU association [14]: G3-4 adverse events 25 vs 37 vs 38\%; febrile neutropenia 2, 14, and 9\%, respectively. Common G3-4 toxicities were: fatigue $21 \%$, neurotoxicity $14 \%$, diarrhea $13 \%$.

Neutropenia was the most common G3-4 toxicity (41\%) with DOC triplet association, DTX $60 \mathrm{mg} / \mathrm{m}^{2}$, OXP $100 \mathrm{mg} / \mathrm{m}^{2} \mathrm{~d} 1$, and capecitabine $500 \mathrm{mg} / \mathrm{m}^{2} /$ bidie, every 3 
Table 3: Cumulative toxicity

\begin{tabular}{|c|c|c|c|c|c|c|c|c|}
\hline \multirow{3}{*}{$\begin{array}{l}\text { Number } \\
\text { NCI-CTC Grade }\end{array}$} & \multicolumn{4}{|c|}{ Patients } & \multicolumn{4}{|c|}{ Cycles } \\
\hline & \multicolumn{4}{|c|}{10} & \multicolumn{4}{|c|}{50} \\
\hline & 1 & 2 & 3 & 4 & 1 & 2 & 3 & 4 \\
\hline Nausea $(\%)$ & $5(50)$ & $1(10)$ & - & - & $11(22)$ & $1(2)$ & - & - \\
\hline Vomiting $(\%)$ & $1(10)$ & $1(10)$ & - & - & $2(4)$ & $1(2)$ & - & - \\
\hline Diarrhea $(\%)$ & $4(40)$ & $4(40)$ & - & - & $12(24)$ & $5(10)$ & - & - \\
\hline Hypoalbuminemia (\%) & - & $3(30)$ & $1(10)$ & - & - & $4(8)$ & $1(2)$ & - \\
\hline Constipation (\%) & $4(40)$ & $2(20)$ & - & - & $9(18)$ & $2(4)$ & - & - \\
\hline Mucositis (\%) & $4(40)$ & $1(10)$ & $1(10)$ & - & $8(16)$ & $2(4)$ & $1(2)$ & - \\
\hline Anorexia (\%) & $2(20)$ & $3(30)$ & - & - & $6(12)$ & $3(6)$ & - & - \\
\hline Asthenia $(\%)$ & $2(20)$ & $6(60)$ & $2(20)$ & - & $16(32)$ & $13(26)$ & $2(4)$ & - \\
\hline Neurotoxicity (\%) & $7(70)$ & $3(30)$ & - & - & $31(62)$ & $4(8)$ & - & - \\
\hline Hypertension (\%) & - & - & - & - & - & - & - & - \\
\hline Hypotension (\%) & - & - & - & - & - & - & - & - \\
\hline Hematuria (\%) & - & - & - & - & - & - & - & - \\
\hline Gengival recession/gengivitis $(\%)$ & - & - & - & - & - & - & - & - \\
\hline Rhinitis (\%) & $1(10)$ & $1(10)$ & - & - & $5(10)$ & $1(2)$ & - & - \\
\hline Epistaxis $(\%)$ & $2(20)$ & - & - & - & $4(8)$ & - & - & - \\
\hline Hand-foot skin reaction $(\%)$ & $2(20)$ & - & - & - & $2(4)$ & - & - & - \\
\hline Hypokalemia (\%) & $2(20)$ & - & - & - & $3(6)$ & - & - & - \\
\hline Hypertransaminasemy (\%) & $2(20)$ & - & - & - & $2(4)$ & - & - & - \\
\hline Hyperpigmentation (\%) & $2(20)$ & - & - & - & $2(4)$ & - & - & - \\
\hline Fever without infection (\%) & - & - & - & - & - & - & - & - \\
\hline Alopecia $(\%)$ & $2(20)$ & $3(30)$ & - & - & $7(14)$ & $3(6)$ & - & - \\
\hline Anemia (\%) & $4(40)$ & $1(10)$ & - & - & $5(10)$ & $1(2)$ & - & - \\
\hline Leucopenia (\%) & $2(20)$ & $3(30)$ & $1(10)$ & $1(10)$ & $7(14)$ & $8(16)$ & $1(2)$ & $1(2)$ \\
\hline Neutropenia (\%) & - & $3(30)$ & $4(40)$ & $1(10)$ & $4(8)$ & $9(18)$ & $6(12)$ & $3(6)$ \\
\hline Thrombocytopenia (\%) & - & $1(10)$ & - & - & - & $2(4)$ & - & - \\
\hline
\end{tabular}

Abbreviation: NCI-CTC, National Cancer Institute Common Toxicity Criteria.

weeks, reaching ORR 52.1\%, PFS 6.9 months, OS 12.6 months [13].

More, different clinical trials evaluated CPT-11 in advanced GC. CPT-11/5-FU was associated with a better safety profile, less treatment discontinuation rate (10.0 vs $21.5 \%$ ), less frequent neutropenia, thrombocytopenia and mucositis, but not diarrhea [16]. In a randomized phase II trial, capecitabine $1000 \mathrm{mg} / \mathrm{m}^{2}$, twice daily for 14 days, was added to CPT-11 $250 \mathrm{mg} / \mathrm{m}^{2}$ or cisplatin $80 \mathrm{mg} / \mathrm{m}^{2}$ $\mathrm{d} 1$, every 3 weeks: prevalent G3-4 toxicities in platinum regimen were thrombocytopenia (18.2 vs $1.8 \%)$, nausea (23.6 vs $12.3 \%)$ and vomiting (16.4 vs $1.8 \%$ ), in CPT-11 arm diarrhea (22.8 vs 7.3\%) [17]. In French trail, FOLFIRI was better tolerated than ECX (overall G3-3 toxicity rate, 69 vs $84 \%$; $\mathrm{P}<0.001)$ [18].

More recently, in the SPIRITS trial, S-1 (40-60 $\mathrm{mg}$ ), twice daily for 3 weeks, plus cisplatin $60 \mathrm{mg} / \mathrm{m}^{2} \mathrm{~d} 8$, followed by 2-week rest, was compared with S-1 for 4 weeks, followed by a 2-week rest [19]. G3-4 leucopenia, neutropenia, anemia, nausea, and anorexia, were more frequent in S-1/cisplatin arm. In west population, cisplatin $75 \mathrm{mg} / \mathrm{m}^{2} \mathrm{~d} 1$ and S-1 $25 \mathrm{mg} / \mathrm{m}^{2}$ bid d1-21, every 28 days, determined G3-4 fatigue (26\%), neutropenia (26\%), vomiting (17\%), diarrhea (15\%), nausea (15\%) [20]. In 


\begin{tabular}{|c|c|c|c|c|}
\hline & \multicolumn{2}{|c|}{$\begin{array}{c}\text { Intent-to-treat } \\
\text { Analysis }\end{array}$} & \multicolumn{2}{|c|}{$\begin{array}{c}\text { As-treated } \\
\text { Analysis }\end{array}$} \\
\hline & No & $\%$ & No & $\%$ \\
\hline Enrolled patients & 10 & 100 & 10 & 100 \\
\hline Evaluable patients & 10 & 100 & 10 & 100 \\
\hline Objective Response & 6 & $60(\mathrm{CI} \pm 32)$ & 6 & $60(\mathrm{CI} \pm 32)$ \\
\hline Partial Response & 5 & 50 & 5 & 50 \\
\hline Complete Response & 1 & 10 & 1 & 10 \\
\hline Stable Disease & 3 & 30 & 3 & 30 \\
\hline Progressive Disease & 1 & 10 & 1 & 10 \\
\hline Median Progression-free survival, months & 6 & & & \\
\hline Range & $3-15$ & 90 & & \\
\hline Progression events & 9 & & & \\
\hline Median Overall Survival, months & 17 & & & \\
\hline Range & $5+-26$ & 70 & & \\
\hline Deaths & 7 & & & \\
\hline
\end{tabular}

FLAGS trial, S-1 $50 \mathrm{mg} / \mathrm{m}^{2} \mathrm{~d} 1-21$ plus cisplatin $75 \mathrm{mg} / \mathrm{m}^{2}$ d 1, every 28 days, more frequent G3-4 toxicities included asthenia (24\%), vomiting (17\%), nausea (15\%), diarrhea (13\%), and neutropenia (19\%) [21, 22]. Less frequent G3-4 toxicities were observed in cisplatin/S-1 compared with cisplatin/5-FU arm: neutropenia (32.3 vs 63.6\%), stomatitis (1.3 vs $13.6 \%$ ), hypokalemia (3.6 vs $10.8 \%$ ). Statistically significant safety advantages were also observed with $\mathrm{S}-1$ : G3-4 neutropenia 18.6 vs $40.0 \%$, febrile neutropenia 1.7 vs $6.9 \%$, mucositis 1.3 vs $13.6 \%$ [23].

The consideration of the present schedule of triplet chemotherapy may also represent a model for integration of different antimetabolite (capecitabine, S-1) and/or different drugs (irinotecan) into the same triplet schedule. Thus, the definition of the proper schedule to safely add DTX to $\mathrm{OXP} / 5-\mathrm{FU}$ doublet chemotherapy represents a mainstay to further investigate in randomized clinical studies whether more intensive first line triplet medical treatments could increase clinical outcome in metastatic GC patients.

Trastuzumab addiction to capecitabine/ cisplatin or 5-FU/cisplatin in HER2 positive patients significantly improved OS up to 16 vs 11.8 months in immunohistochemistry (IHC) $2+$ and fluorescence in-situ hybridization (FISH) positive or IHC3+ [31]. Most common adverse events were: nausea (67 vs $63 \%$ ), vomiting (50 vs 46\%), neutropenia (53 vs 57\%), cardiac adverse events ( $6 \%$ both arms). Definition of a feasible and safe triplet chemotherapy schedule could be potentially developed for a more intensive first line regimen adding trastuzumab in HER2 positive metastatic GC patients.

Second line CPT-11 plus mitomycin-c reported ORR 32\%, PFS 4 months, OS 8 months [32]. PFS and OS were 3.1 and 6.5 months with capecitabine and CPT-11 [33]. Retrospective evaluation of DTX plus CPT-11, after platinum-based therapy reported PFS 11 weeks, OS 24 weeks [34]. In a phase III study, among patients refractory to fluoropyrimidine plus platinum, ORR, PFS and OS were not significantly different in paclitaxel compared to CPT-11: $20.9 \%, 3.6$ and 8.4 months vs $13.6 \%, 2.3$ and 9.5 months, respectively [35]. FOLFIRI in patients who failed DTX-containing first-line therapy reached ORR 22.8\%, PFS 3.8 months and OS 6.2 months [36].

In metastatic $\mathrm{GC}$ patients, progressed after first-line platinum- or fluoropyrimidine-containing chemotherapy, OS was significantly increased to 5.2 vs 3.8 months $P 0.047$, with ramucirumab, $8 \mathrm{mg} / \mathrm{kg}$ every 2 weeks, compared with placebo [37]. Addiction of ramucirumab to paclitaxel, after first-line platinum plus fluoropyrimidine with or without anthracycline, significantly increased OS up to 9.6 vs. 7.4 months, $P 0.017$ [38]. The increasing efficacy of ramucirumab plus taxane as second line treatment point the discussion on the possibility to adopt a sequential treatment strategy alternative to a more intensive first line regimen in metastatic GC, particularly in patients unfit for such an intensive combined therapy, due to clinical parameters, age, comorbidity, functional/ nutritional conditions.

\section{MATERIALS AND METHODS}

\section{Patient eligibility}

Patients were eligible if they had histologically confirmed diagnosis of measurable metastatic GC; age 18-75 years; World Health Organization (WHO) PS $\leq 2$; 
adequate hematological, renal and hepatic functions; life expectancy more than 3 months.

CIRS was used to evaluate the comorbidity status, and only patients with primary and intermediate CIRS stage were enrolled [30]. Primary CIRS stage consisted of: independent Instrumental Activity of Daily Living (IADL), and absent or mild grade comorbidities; intermediate CIRS stage consisted of dependent or independent IADL, and $<3$ mild or moderate grade comorbidities. Patients with secondary CIRS stage, consisting of $\geq 3$ comorbidities or a severe comorbidity, with or without dependent IADL, were not enrolled. Criteria to define patients unfit for the proposed treatment strategy were: uncontrolled severe diseases; cardiovascular disease (uncontrolled hypertension, uncontrolled arrhythmia, ischemic cardiac diseases in the last year); thromboembolic disease, coagulopathy, preexisting bleeding diatheses.

First line DTX association to 5-FU and OXP, was proposed to consecutive eligible metastatic GC patients as a treatment strategy in clinical practice, chosen among those in indication and approved by Agenzia Italiana del Farmaco (AIFA) for administration in label in Italian public hospitals, and published in Gazzetta Ufficiale Repubblica Italiana ("Elenco dei Medicinali erogabili a totale carico del Servizio Sanitario Nazionale", Gazzetta Ufficiale Repubblica Italiana N.1, 2 Gennaio 2009). Thus, it was not necessary any approval by ethics committee and institutional review board, because patients were treated with conventional treatments without any additional medical intervention out of the best common clinical practice. All patients provided written, informed consent to the proposed in label treatment strategy. Treatment was conducted in accordance with the Declaration of Helsinki.

\section{Methods}

\section{Schedule}

It was a dose-finding study evaluating safety and activity of triplet chemotherapy association, consisting of DTX, 5-FU and OXP, as first line treatment in advanced GC patients. Triplet chemotherapy association was administered according to the following schedule: DTX (Taxotere; Sanofi-Aventis, Milan, Italy), administered over 60 minutes as intravenous infusion in $250 \mathrm{ml}$ of $\mathrm{NaCl} 0.9 \%$, at the dose of $50 \mathrm{mg} / \mathrm{m}^{2} \mathrm{~d} 1,15$; TFI $/ 5-\mathrm{FU}$ (Fluorouracil Teva $^{\circledR}$, Teva), 700-800-900-1000 mg/m²/die, over 12-hour (from 10:00 p.m to 10:00 a.m.), d1-2, 8-9, 15-16 and 22-23; OXP (Eloxatin; Sanofi-Aventis, Milan, Italy), over 2-hours as an intravenous infusion in $250 \mathrm{ml}$ of dextrose $5 \%$, at the dose of $60-70-80 \mathrm{mg} / \mathrm{m}^{2} \mathrm{~d} 8,22$. Cycles repeated every 4 weeks. 5-FU was administered by a portable pump (CADD Plus, SEVIT) using a venous access device.

\section{Study design}

Physical examination and routine laboratory tests were performed at baseline and every week on-treatment, including complete blood cell count, electrolytes, liver and renal function, urine examination and coagulation function; tumor markers every 4 weeks; electrocardiogram every cycle and echocardiogram at baseline, and every 3 cycles of treatment.

Primary end-point was to define the recommended 5-FU and OXP doses. Secondary end-points were evaluation of toxicity, ORR, PFS, OS. Toxicity was registered every week according to National Cancer Institute Common Toxicity Criteria (version 3.0). DLT was defined: in the intra-patient step, as G2 non-haematological or G3 haematological toxicity; in the inter-patient step, as grade 3-4 non-haematological toxicity (mainly represented by diarrhea, mucositis, neurotoxicity, hand-foot syndrome, asthenia), grade 4 hematologic toxicity (neutropenia), febrile neutropenia, grade 3-4 thrombocytopenia, or any toxicity determining $>2$ weeks treatment delay.

To discriminate individual safety, LTS, consisting of at least a limiting toxicity (LT) associated or not to other limiting or G2 toxicities, were evaluated, as previously reported $[25,28]$. LTS were classified as LTS single site (LTS-ss), characterized only by the LT, and LTS multiple sites (LTS-ms), $\geq 2$ LTs or a LT associated to other, at least $\mathrm{G} 2$, non-limiting toxicities.

ORR was evaluated according to RECIST criteria [39]; pathologic complete response was defined as absence of residual cancer cells in surgically resected specimens; PFS and OS, using Kaplan-Meier method [40]. PFS was defined as length of time between the beginning of treatment and disease progression or death (resulting from any cause) or to last contact; OS as length of time between the beginning of treatment and death or to last contact.

Patients were evaluated at baseline and after treatment by a multidisciplinary team, consisting of medical oncologist, radiotherapist, surgeon, and radiologist, to dynamically evaluate multimodality treatment strategy. Surgical resection was defined $\mathrm{R} 0$, if radical surgery, $\mathrm{R} 1$, if microscopic residual cancer cells were present at resection margins. Surgery was recommended > 4-6 weeks after chemotherapy discontinuation.

Clinical evaluation of response was planned by CTscan; PET and MRI were added based on investigators' assessment; response on primary gastric tumor was also evaluated by endoscopy. Follow-up was scheduled every three months up to disease progression or death.

\section{Statistical design}

This dose-finding study was developed to verify recommended OXP dose, by 3 escalating dose steps at 60 , 
70 and $80 \mathrm{mg} / \mathrm{m}^{2}$, and 5-FU dose, by 4 escalating dose steps at $700,800,900$ and $1000 \mathrm{mg} / \mathrm{m}^{2} / \mathrm{d}$, according to an intra- and inter-patient approach $[41,42]$. It is preliminary to a phase II study, evaluating activity and efficacy of triplet chemotherapy association, assuming as minimal interesting activity a rate of $40 \%$, according to Simon two stage design [42].

\section{CONCLUSIONS}

The present dose-finding study proposed a feasible and safe schedule of triplet DTX/5-FU/OXP association, $\mathrm{FD} / \mathrm{FOx}$ regimen, at 5-FU and OXP recommended doses $1000 \mathrm{mg} / \mathrm{m}^{2} / \mathrm{d}$ and $80 \mathrm{mg} / \mathrm{m}^{2}$, respectively, that should be evaluated in prospective trials as first line treatment in metastatic GC patients.

\section{Abbreviations}

CIRS: Cumulative Illness Rating Scale; CF: cisplatin, 5-fluorouracil; CPT-11: irinotecan; DCF: docetaxel, cisplatin, 5-fluorouracil; DCR: disease control rate; DI: dose intensity; DLT: dose-limiting toxicity; DTX: docetaxel; ECF: epirubicin, cisplatin, 5-fluorouracil; ECX: epirubicin, cisplatin, capecitabine; EOF: epirubicin, oxaliplatin, 5-fluorouracil; EOX: epirubicin, oxaiplatin, capecitabine; FAM: 5-FU, doxorubicin, mitomycin; FLO: 5-FU, leucovorin, oxaliplatin; FOLFIRI: 5-fluorouracil, leucovorin, irinotecan; FP: cisplatin, 5-fluorouracil; GC, gastric cancer; IADL: Instrumental Activity of Daily Living; LTS: limiting toxicity syndromes; LTSms: limiting toxicity syndromes multiple sites; MCF: mitomycin, cisplatin, 5-fluorouracil; mDCF: modified docetaxel, cisplatin, 5-fluorouracil; MTD: maximum tolerated dose; ORR: objective response rate; OS: overall survival; OXP: oxaliplatin; PFS: progression-free survival; PS, performance status; QoL: quality of life; RD: recommended dose; rDI: received dose intensity; WHO: World Health Organization; XP: cisplatin, capecitabine; 5-FU: 5-fluorouracil.

\section{Author contributions}

GB contributed to the conception and design of the study, in the provision of study materials of patients, in the clinical management, in the data analysis and interpretation, in the manuscript writing. ER contributed to the conception and design of the study, in clinical management, in the data analysis and interpretation, in the manuscript writing. SM, EC contributed in schedule designing. AG, AR contributed in the data interpretation. ADM, GC provided biological analysis. SG provided surgical evaluations. All authors participated in the collection and/or assembly of data. All authors read, revised and approved the final manuscript.

\section{CONFLICTS OF INTEREST}

Authors declare that they have no conflicts of interest.

\section{FUNDING}

None.

\section{REFERENCES}

1. Cullinan SA, Moertel CG, Fleming TR, Rubin JR, Krook JE, Everson LK, Windschitl HE, Twito DI, Marschke RF, Foley JF, Pfeifle DM, Barlow JF. A comparison of three chemotherapeutic regimens in the treatment of advanced pancreatic and gastric carcinoma. Fluorouracil vs fluorouracil and doxorubicin vs fluorouracil, doxorubicin, and mitomycin. JAMA. 1985; 253:2061-67. https://doi. org/10.1001/jama.1985.03350380077025.

2. Webb A, Cunningham D, Scarffe JH, Harper P, Norman A, Joffe JK, Hughes M, Mansi J, Findlay M, Hill A, Oates J, Nicolson M, Hickish T, et al. Randomized trial comparing epirubicin, cisplatin, and fluorouracil versus fluorouracil, doxorubicin, and methotrexate in advanced esophagogastric cancer. J Clin Oncol. 1997; 15:261-67. https://doi. org/10.1200/JCO.1997.15.1.261.

3. Ross P, Nicolson M, Cunningham D, Valle J, Seymour M, Harper P, Price T, Anderson H, Iveson T, Hickish T, Lofts F, Norman A. Prospective randomized trial comparing mitomycin, cisplatin, and protracted venous-infusion fluorouracil (PVI 5-FU) With epirubicin, cisplatin, and PVI 5-FU in advanced esophagogastric cancer. J Clin Oncol. 2002; 20:1996-2004. https://doi.org/10.1200/ JCO.2002.08.105.

4. Louvet C, André T, Tigaud JM, Gamelin E, Douillard JY, Brunet R, François E, Jacob JH, Levoir D, Taamma A, Rougier P, Cvitkovic E, de Gramont A. Phase II study of oxaliplatin, fluorouracil, and folinic acid in locally advanced or metastatic gastric cancer patients. J Clin Oncol. 2002; 20:4543-48. https://doi.org/10.1200/JCO.2002.02.021.

5. Al-Batran SE, Hartmann JT, Probst S, Schmalenberg H, Hollerbach S, Hofheinz R, Rethwisch V, Seipelt G, Homann N, Wilhelm G, Schuch G, Stoehlmacher J, Derigs HG, et al, and Arbeitsgemeinschaft Internistische Onkologie. Phase III trial in metastatic gastroesophageal adenocarcinoma with fluorouracil, leucovorin plus either oxaliplatin or cisplatin: a study of the Arbeitsgemeinschaft Internistische Onkologie. J Clin Oncol. 2008; 26:1435-42. https://doi.org/10.1200/ JCO.2007.13.9378.

6. Cunningham D, Starling N, Rao S, Iveson T, Nicolson M, Coxon F, Middleton G, Daniel F, Oates J, Norman AR, and Upper Gastrointestinal Clinical Studies Group of the National Cancer Research Institute of the United Kingdom. Capecitabine and oxaliplatin for advanced esophagogastric 
cancer. N Engl J Med. 2008; 358:36-46. https://doi. org/10.1056/NEJMoa073149.

7. Kang YK, Kang WK, Shin DB, Chen J, Xiong J, Wang J, Lichinitser M, Guan Z, Khasanov R, Zheng L, Philco-Salas M, Suarez T, Santamaria J, et al. Capecitabine/cisplatin versus 5-fluorouracil/cisplatin as first-line therapy in patients with advanced gastric cancer: a randomised phase III noninferiority trial. Ann Oncol. 2009; 20:666-73. https:// doi.org/10.1093/annonc/mdn717.

8. Okines AF, Norman AR, McCloud P, Kang YK, Cunningham D. Meta-analysis of the REAL-2 and ML17032 trials: evaluating capecitabine-based combination chemotherapy and infused 5-fluorouracil-based combination chemotherapy for the treatment of advanced oesophago-gastric cancer. Ann Oncol. 2009; 20:1529-34. https://doi.org/10.1093/annonc/mdp047.

9. Van Cutsem E, Moiseyenko VM, Tjulandin S, Majlis A, Constenla M, Boni C, Rodrigues A, Fodor M, Chao Y, Voznyi E, Risse ML, Ajani JA, and V325 Study Group. Phase III study of docetaxel and cisplatin plus fluorouracil compared with cisplatin and fluorouracil as first-line therapy for advanced gastric cancer: a report of the V325 Study Group. J Clin Oncol. 2006; 24:4991-97. https://doi. org/10.1200/JCO.2006.06.8429.

10. Roth AD, Fazio N, Stupp R, Falk S, Bernhard J, Saletti P, Köberle D, Borner MM, Rufibach K, Maibach R, Wernli M, Leslie M, Glynne-Jones R, et al, and Swiss Group for Clinical Cancer Research. Docetaxel, cisplatin, and fluorouracil; docetaxel and cisplatin; and epirubicin, cisplatin, and fluorouracil as systemic treatment for advanced gastric carcinoma: a randomized phase II trial of the Swiss Group for Clinical Cancer Research. J Clin Oncol. 2007; 25:3217-23. https://doi.org/10.1200/ JCO.2006.08.0135

11. Al-Batran SE, Hartmann JT, Hofheinz R, Homann N, Rethwisch V, Probst S, Stoehlmacher J, Clemens MR, Mahlberg R, Fritz M, Seipelt G, Sievert M, Pauligk C, et al. Biweekly fluorouracil, leucovorin, oxaliplatin, and docetaxel (FLOT) for patients with metastatic adenocarcinoma of the stomach or esophagogastric junction: a phase II trial of the Arbeitsgemeinschaft Internistische Onkologie. Ann Oncol. 2008; 19:1882-87. https://doi.org/10.1093/annonc/mdn403.

12. Inal A, Kaplan MA, Kucukoner M, Isikdogan A. Docetaxel and Cisplatin Plus Fluorouracil compared with Modified Docetaxel, Cisplatin, and 5-Fluorouracil as first-line therapy for advanced gastric cancer: a retrospective analysis of single institution. Neoplasma. 2012; 59:233-36. https://doi. org/10.4149/neo_2012_030.

13. Di Lauro L, Vici P, Belli F, Tomao S, Fattoruso SI, Arena MG, Pizzuti L, Giannarelli D, Paoletti G, Barba M, Sergi D, Maugeri-Saccà M. Docetaxel, oxaliplatin, and capecitabine combination chemotherapy for metastatic gastric cancer. Gastric Cancer. 2014; 17:718-24. https://doi.org/10.1007/ s10120-013-0321-3.
14. Van Cutsem E, Boni C, Tabernero J, Massuti B, Middleton G, Dane F, Reichardt P, Pimentel FL, Cohn A, Follana P, Clemens M, Zaniboni A, Moiseyenko V, et al. Docetaxel plus oxaliplatin with or without fluorouracil or capecitabine in metastatic or locally recurrent gastric cancer: a randomized phase II study. Ann Oncol. 2015; 26:149-56. https://doi.org/10.1093/annonc/mdu496.

15. Shah MA, Janjigian YY, Stoller R, Shibata S, Kemeny M, Krishnamurthi S, Su YB, Ocean A, Capanu M, Mehrotra B, Ritch P, Henderson C, Kelsen DP. Randomized Multicenter Phase II Study of Modified Docetaxel, Cisplatin, and Fluorouracil (DCF) Versus DCF Plus Growth Factor Support in Patients With Metastatic Gastric Adenocarcinoma: A Study of the US Gastric Cancer Consortium. J Clin Oncol. 2015; 33:3874-79. https://doi. org/10.1200/JCO.2015.60.7465.

16. Dank M, Zaluski J, Barone C, Valvere V, Yalcin S, Peschel C, Wenczl M, Goker E, Cisar L, Wang K, Bugat R. Randomized phase III study comparing irinotecan combined with 5-fluorouracil and folinic acid to cisplatin combined with 5-fluorouracil in chemotherapy naive patients with advanced adenocarcinoma of the stomach or esophagogastric junction. Ann Oncol. 2008; 19:1450-57. https://doi.org/10.1093/annonc/mdn166.

17. Moehler M, Kanzler S, Geissler M, Raedle J, Ebert MP, Daum S, Flieger D, Seufferlein T, Galle PR, Hoehler T, and Arbeitsgemeinschaft Internistische Onkologie, Germany. A randomized multicenter phase II study comparing capecitabine with irinotecan or cisplatin in metastatic adenocarcinoma of the stomach or esophagogastric junction. Ann Oncol. 2010; 21:71-77. https://doi.org/10.1093/ annonc/mdp269.

18. Guimbaud R, Louvet C, Ries P, Ychou M, Maillard E, André T, Gornet JM, Aparicio T, Nguyen S, Azzedine A, Etienne PL, Boucher E, Rebischung C, et al. Prospective, randomized, multicenter, phase III study of fluorouracil, leucovorin, and irinotecan versus epirubicin, cisplatin, and capecitabine in advanced gastric adenocarcinoma: a French intergroup (Fédération Francophone de Cancérologie Digestive, Fédération Nationale des Centres de Lutte Contre le Cancer, and Groupe Coopérateur Multidisciplinaire en Oncologie) study. J Clin Oncol. 2014; 32:3520-26. https:// doi.org/10.1200/JCO.2013.54.1011.

19. Koizumi W, Narahara H, Hara T, Takagane A, Akiya T, Takagi M, Miyashita K, Nishizaki T, Kobayashi O, Takiyama W, Toh Y, Nagaie T, Takagi S, et al. S-1 plus cisplatin versus S-1 alone for first-line treatment of advanced gastric cancer (SPIRITS trial): a phase III trial. Lancet Oncol. 2008; 9:215-21. https://doi.org/10.1016/ S1470-2045(08)70035-4.

20. Ajani JA, Lee FC, Singh DA, Haller DG, Lenz HJ, Benson AB 3rd, Yanagihara R, Phan AT, Yao JC, Strumberg D. Multicenter phase II trial of S-1 plus cisplatin in patients with untreated advanced gastric or gastroesophageal 
junction adenocarcinoma. J Clin Oncol. 2006; 24:663-67. https://doi.org/10.1200/JCO.2005.04.2994.

21. Lenz HJ, Lee FC, Haller DG, Singh D, Benson AB 3rd, Strumberg D, Yanagihara R, Yao JC, Phan AT, Ajani JA. Extended safety and efficacy data on S-1 plus cisplatin in patients with untreated, advanced gastric carcinoma in a multicenter phase II study. Cancer. 2007; 109:33-40. https://doi.org/10.1002/cncr.22329.

22. Ajani JA, Rodriguez W, Bodoky G, Moiseyenko V, Lichinitser M, Gorbunova V, Vynnychenko I, Garin A, Lang I, Falcon S. Multicenter phase III comparison of cisplatin/S-1 with cisplatin/infusional fluorouracil in advanced gastric or gastroesophageal adenocarcinoma study: the FLAGS trial. J Clin Oncol. 2010; 28:1547-53. https://doi.org/10.1200/JCO.2009.25.4706.

23. Ajani JA, Buyse M, Lichinitser M, Gorbunova V, Bodoky G, Douillard JY, Cascinu S, Heinemann V, Zaucha R, Carrato A, Ferry D, Moiseyenko V. Combination of cisplatin/S-1 in the treatment of patients with advanced gastric or gastroesophageal adenocarcinoma: results of noninferiority and safety analyses compared with cisplatin/5-fluorouracil in the First-Line Advanced Gastric Cancer Study. Eur J Cancer. 2013; 49:3616-24. https://doi. org/10.1016/j.ejca.2013.07.003.

24. Morelli MF, Santomaggio A, Ricevuto E, Cannita K, De Galitiis F, Tudini M, Bruera G, Mancini M, Pelliccione M, Calista F, Guglielmi F, Martella F, Lanfiuti Baldi P, et al, and CINBO. Triplet schedule of weekly 5-fluorouracil and alternating irinotecan or oxaliplatin in advanced colorectal cancer: a dose-finding and phase II study. Oncol Rep. 2010; 23:1635-40.

25. Bruera G, Santomaggio A, Cannita K, Baldi PL, Tudini M, De Galitiis F, Mancini M, Marchetti P, Antonucci A, Ficorella C, Ricevuto E. "Poker" association of weekly alternating 5-fluorouracil, irinotecan, bevacizumab and oxaliplatin (FIr-B/FOx) in first line treatment of metastatic colorectal cancer: a phase II study. BMC Cancer. 2010; 10:567. https://doi.org/10.1186/1471-2407-10-567.

26. Bruera G, Ricevuto E. Intensive chemotherapy of metastatic colorectal cancer: weighing between safety and clinical efficacy: Evaluation of Masi G, Loupakis F, Salvatore L, et al. Bevacizumab with FOLFOXIRI (irinotecan, oxaliplatin, fluorouracil, and folinate) as first-line treatment for metastatic colorectal cancer: a phase 2 trial. Lancet Oncol 2010;11:845-52. Expert Opin Biol Ther. 2011; 11:821-24. https://doi.org/10.1517/14712598.2011.582462.

27. Ficorella C, Bruera G, Cannita K, Porzio G, Lanfiuti Baldi P, Tinari N, Natoli C, Ricevuto E. Triplet chemotherapy in patients with metastatic colorectal cancer: toward the best way to safely administer a highly active regimen in clinical practice. Clin Colorectal Cancer. 2012; 11:229-37. https:// doi.org/10.1016/j.clcc.2012.05.001.

28. Bruera G, Cannita K, Giordano AV, Vicentini R, Ficorella C, Ricevuto E. Effectiveness and safety of intensive triplet chemotherapy plus bevacizumab, FIr-B/FOx, in young-elderly metastatic colorectal cancer patients. Biomed Res Int. 2013; 2013:143273. https://doi. org/10.1155/2013/143273.

29. Bruera G, Russo A, Galvano A, Rizzo S, Ricevuto E. Clinical parameters to guide decision-making in elderly metastatic colorectal CANCER patients treated with intensive cytotoxic and anti-angiogenic therapy. Oncotarget. 2017; 8:37875-83. https://doi.org/10.18632/ oncotarget.14333.

30. Extermann M, Overcash J, Lyman GH, Parr J, Balducci L. Comorbidity and functional status are independent in older cancer patients. J Clin Oncol. 1998; 16:1582-87. https://doi. org/10.1200/JCO.1998.16.4.1582.

31. Bang YJ, Van Cutsem E, Feyereislova A, Chung HC, Shen L, Sawaki A, Lordick F, Ohtsu A, Omuro Y, Satoh T, Aprile G, Kulikov E, Hill J, et al, and ToGA Trial Investigators. Trastuzumab in combination with chemotherapy versus chemotherapy alone for treatment of HER2-positive advanced gastric or gastro-oesophageal junction cancer (ToGA): a phase 3, open-label, randomised controlled trial. Lancet. 2010; 376:687-97. https://doi.org/10.1016/ S0140-6736(10)61121-X.

32. Giuliani F, Molica S, Maiello E, Battaglia C, Gebbia V, Di Bisceglie M, Vinciarelli G, Gebbia N, Colucci G, and Gruppo Oncologico dell' Italia Meridionale (prot. 2106). Irinotecan (CPT-11) and mitomycin-C (MMC) as secondline therapy in advanced gastric cancer: a phase II study of the Gruppo Oncologico dell' Italia Meridionale (prot. 2106). Am J Clin Oncol. 2005; 28:581-85. https://doi. org/10.1097/01.coc.0000190398.52142.7f.

33. Leary A, Assersohn L, Cunningham D, Norman AR, Chong G, Brown G, Ross PJ, Costello C, Higgins L, Oates J. A phase II trial evaluating capecitabine and irinotecan as second line treatment in patients with oesophago-gastric cancer who have progressed on, or within 3 months of platinum-based chemotherapy. Cancer Chemother Pharmacol. 2009; 64:455-62. https://doi.org/10.1007/ s00280-008-0893-5.

34. Hawkes E, Okines AF, Papamichael D, Rao S, Ashley S, Charalambous H, Koukouma A, Chau I, Cunningham D. Docetaxel and irinotecan as second-line therapy for advanced oesophagogastric cancer. Eur J Cancer. 2011; 47:1146-51. https://doi.org/10.1016/j.ejca.2010.12.021.

35. Hironaka S, Ueda S, Yasui H, Nishina T, Tsuda M, Tsumura T, Sugimoto N, Shimodaira H, Tokunaga S, Moriwaki T, Esaki T, Nagase M, Fujitani K, et al. Randomized, openlabel, phase III study comparing irinotecan with paclitaxel in patients with advanced gastric cancer without severe peritoneal metastasis after failure of prior combination chemotherapy using fluoropyrimidine plus platinum: WJOG 4007 trial. J Clin Oncol. 2013; 31:4438-44. https:// doi.org/10.1200/JCO.2012.48.5805.

36. Maugeri-Saccà M, Pizzuti L, Sergi D, Barba M, Belli F, Fattoruso S, Giannarelli D, Amodio A, Boggia S, Vici P, Di Lauro L. FOLFIRI as a second-line therapy in patients 
with docetaxel-pretreated gastric cancer: a historical cohort. J Exp Clin Cancer Res. 2013; 32:67. https://doi. org/10.1186/1756-9966-32-67.

37. Fuchs CS, Tomasek J, Yong CJ, Dumitru F, Passalacqua R, Goswami C, Safran H, Dos Santos LV, Aprile G, Ferry DR, Melichar B, Tehfe M, Topuzov E, et al, and REGARD Trial Investigators. Ramucirumab monotherapy for previously treated advanced gastric or gastro-oesophageal junction adenocarcinoma (REGARD): an international, randomised, multicentre, placebo-controlled, phase 3 trial. Lancet. 2014; 383:31-39. https://doi.org/10.1016/ S0140-6736(13)61719-5.

38. Wilke H, Muro K, Van Cutsem E, Oh SC, Bodoky G, Shimada Y, Hironaka S, Sugimoto N, Lipatov O, Kim TY, Cunningham D, Rougier P, Komatsu Y, et al, and RAINBOW Study Group. Ramucirumab plus paclitaxel versus placebo plus paclitaxel in patients with previously treated advanced gastric or gastro-oesophageal junction adenocarcinoma (RAINBOW): a double-blind, randomised phase 3 trial. Lancet Oncol. 2014; 15:1224-35. https://doi. org/10.1016/S1470-2045(14)70420-6.

39. Therasse P, Arbuck SG, Eisenhauer EA, Wanders J, Kaplan RS, Rubinstein L, Verweij J, Van Glabbeke M, van Oosterom AT, Christian MC, Gwyther SG. New guidelines to evaluate the response to treatment in solid tumors. European Organization for Research and Treatment of Cancer, National Cancer Institute of the United States, National Cancer Institute of Canada. J Natl Cancer Inst. 2000; 92:205-16. https://doi.org/10.1093/jnci/92.3.205.

40. Kaplan EL, Meier P. Nonparametric estimation of incomplete observations. J Am Stat Assoc. 1958; 53:45781. https://doi.org/10.1080/01621459.1958.10501452.

41. Simon R, Freidlin B, Rubinstein L, Arbuck SG, Collins J, Christian MC. Accelerated titration designs for phase I clinical trials in oncology. J Natl Cancer Inst. 1997; 89:1138-47. https://doi.org/10.1093/jnci/89.15.1138.

42. Simon R. Optimal two-stage designs for phase II clinical trials. Control Clin Trials. 1989; 10:1-10. https://doi. org/10.1016/0197-2456(89)90015-9. 